\title{
Protocolo para Disseminação de Dados em VANETs baseado em Métricas de Redes Complexas: Um Estudo de Caso com Sistema de Gerenciamento de Trânsito
}

\author{
Joahannes Costa ${ }^{1,2}$, Denis Rosário ${ }^{1}$, Allan M. de Souza ${ }^{2}$, \\ Leandro A. Villas $^{2}$, Eduardo Cerqueira ${ }^{1}$ \\ ${ }^{1}$ Universidade Federal do Pará (UFPA), Belém - Pará - Brasil \\ \{joahannes, denis, cerqueira\}@ufpa.br \\ ${ }^{2}$ Instituto de Computação (UNICAMP), Campinas - São Paulo - Brasil \\ allanmselrc.ic.unicamp.br, leandro@ic.unicamp.br
}

\begin{abstract}
Several services in Vehicular Ad-Hoc Networks (VANETs), such as Traffic Management Systems (TMS), require disseminated information among vehicles for efficient decision-making. However, data dissemination is a challenging task due to the specific characteristics of VANETs, such as heterogeneous density, short-range communication, and high node mobility. In this paper, we introduce a data dissemination protocol for urban scenarios that considers complex network metrics, called DDRX. The simulation results show that DDRX offers high coverage, lower number of transmissions and delay compared to other well-known dissemination protocols. In addition, when applied to a TMS, it allows vehicles to stay less time in congestion, have less travel time and reach higher speeds.
\end{abstract}

Resumo. Diversos serviços em Redes Veiculares Ad-Hoc (VANETs), tal como Sistemas de Gerenciamento de Trânsito (TMS), requerem informações disseminadas entre os veículos para tomada de decisões. Contudo, disseminação de dados é uma tarefa desafiadora, devido às características específicas das VANETs, tais como, densidade heterogênea, comunicação de curto alcance e alta mobilidade dos nós. Neste artigo é apresentado um protocolo para disseminação de dados em cenários urbanos que considera métricas de redes complexas, denominado DDRX. Os resultados de simulações mostram que o DDRX oferece alta eficiência em termos de cobertura, número de transmissões e atraso em comparação ao protocolos amplamente conhecidos de disseminação de dados. Além disso, quando aplicado a um TMS, possibilita que os veículos fiquem menos tempo em congestionamentos, tenham menor tempo de viagem e atinjam maiores velocidades.

\section{Introdução}

As Redes Veiculares Ad-Hoc (VANETs) prometem um amplo escopo de serviços que vão desde segurança, assistência aos motoristas e entretenimento [Gerla et al. 2014]. As VANETs são compostas de veículos em movimento com sensores integrados, unidades de processamento e armazenamento, além de interfaces de comunicação sem fio, onde esses veículos podem cooperar entre si para criar uma rede ad-hoc, fornecendo e recebendo dados de outras entidades [de Souza and Villas 2016]. Neste contexto, as VANETs permitem a comunicação tanto de veículos para veículos (vehicle-to-vehicle - V2V) quanto de veículos para uma infraestrutura (vehicle-to-infrastructure - V2I) [Wang et al. 2015]. 
Um Sistema de Gerenciamento de Trânsito (Traffic Management Systems - TMS) necessita de dados que descrevam os padrões de tráfego, tais como, densidade, velocidade, tempo de viagem e posição geográfica dos veículos [de Souza et al. 2016]. Tais informações devem ser disseminadas pela VANET para serem entregues ao TMS, o qual realiza a administração do fluxo de tráfego de veículos, planejamento de caminho individualizado, localização de veículos, dentre outros [Lv et al. 2015, Wang et al. 2015]. No entanto, a disseminação de dados de forma eficiente com baixo overhead e atraso, bem como alta cobertura não é uma tarefa trivial, uma vez que as VANETs possuem uma densidade dinâmica, falhas na comunicação V2V devido a alta mobilidade, além da comunicação de curto alcance [Silva et al. 2016].

A disseminação de dados por meio da inundação da rede, conhecida como Flooding, é a abordagem mais simples para disseminar dados a partir de uma fonte para todos os veículos localizados dentro de uma Área de Interesse (AoI). Nessa abordagem, o veículo fonte inicia a transmissão da mensagem para todos os veículos no seu alcance, e cada veículo alcançado também repete esse processo. As retransmissões ocorrem sucessivamente até que a rede seja inundada com a mensagem. O Flooding tem um bom desempenho em VANETs com baixa densidade. Porém, essa abordagem não trata do problema de broadcast storm [Akabane et al. 2016] que causa sobrecarga na rede, esse problema é muito comum em cenários densos, devido as transmissões de mensagens redundantes ou múltiplas transmissões em um curto intervalo de tempo. Portanto, os protocolos de disseminação de dados em VANETs devem lidar com o problema de broadcast storm, elevados atrasos, baixa cobertura e colisões de pacotes [Cunha et al. 2016].

Disseminar dados com base no conhecimento contextual além dos vizinhos a 1 salto provê uma seleção eficiente de nós encaminhadores. Especificamente, os dados podem ser encaminhados para o veículo identificado como um ponto comum de comunicação a partir de uma análise topológica, e.g., um nó que tenha maior número de vizinhos. Nesse contexto, a rede pode ser representada através de um grafo, onde os veículos são considerados como vértices e os enlaces de comunicação entre eles como arestas. Com base nesse grafo é possível analisar o comportamento da rede a partir de métricas de redes complexas [Zhang and Li 2015]. Redes complexas permitem modelar estruturas e aspectos do mundo real através de elementos e conexões entre os elementos para resolução de problemas específicos, ou seja, por meio de grafos [Newman 2003]. Modelar as VANETs como grafos permite avaliar as interações entre veículos e como elas impactam na rede como um todo [Alvarenga et al. 2014, Wang et al. 2017].

Este artigo apresenta o DDRX (Disseminação de Dados baseada em métricas de Redes compleXas), um protocolo distribuído que permite disseminar dados com baixo overhead e atraso, maximizando a cobertura. Para isso, cada veículo deve conhecer sobre seus vizinhos, a fim de identificar o melhor veículo para continuar o processo de disseminação, baseado nas métricas de centralidade de intermediação (betweenness centrality) e centralidade de grau (degree centrality).

Os resultados mostraram que o DDRX é capaz de reduzir a quantidade de transmissões, atraso e colisões em $65.09 \%, 64.03 \%$ e $55.89 \%$, quando comparado a outros protocolos da literatura. Além disso, um estudo de caso utilizando um TMS mostrou que a utilização do DDRX é capaz de aumentar sua eficiência, melhorando as condições de tráfego e também utilização da rede. Em particular, utilizando o DDRX o sistema é capaz de reduzir a quantidade de transmissões em 95\%, reduzir o tempo de congestionamento em $48.95 \%$ e manter o tempo de viagem próximo das outras soluções. 
O restante deste artigo é organizado da seguinte forma. A Seção 2 apresenta os trabalhos relacionados. A Seção 3 apresenta o protocolo de disseminação de dados DDRX. A Seção 4 descreve o cenário de avaliação e os resultados obtidos. Na Seção 5 é apresentado um estudo de caso aplicando o DDRX em um TMS, bem como os resultados obtidos. Por fim, a Seção 6 apresenta as conclusões e perspectivas futuras.

\section{Trabalhos Relacionados}

Viriyasitavat et al. [Viriyasitavat et al. 2010] propuseram o protocolo Urban Vehicular broadCAST (UV-CAST), o qual concentra-se na disseminação de dados em VANETs tanto em cenários densos quanto esparsos. No UV-CAST, cada veículo pode operar em um dos dois estados: supressão de broadcast ou store-carry-forward. Quando um veículo recebe uma mensagem pela primeira vez, ele verifica se é um veículo de borda ou não, ou seja, aqueles que estão na fronteira de um componente conectado. O UV-CAST assume que esses veículos têm uma maior probabilidade de conhecer novos vizinhos e, portanto, eles armazenam a mensagem e carregam-na até encontrar um novo vizinho. Por outro lado, se o veículo não é um veículo de fronteira, ele executa um algoritmo de supressão de broadcast para retransmitir a mensagem. No entanto, o UV-CAST apresenta um alto overhead, uma vez que cada veículo tem que transmitir o pacote em cada contato com veículos vizinhos que ainda não receberam esse pacote.

Meneguette et al. [Meneguette et al. 2014] expuseram o ALgoritmo Autônomo para Disseminação De INformações em Redes Veiculares (ALADDIN), o qual tem como principal objetivo diminuir o número de retransmissões de uma mensagem de dados, sem comprometer a cobertura. Utiliza zonas de preferência para mitigar o problema de broadcast storm, que é uma área onde os veículos em seu interior são considerados mais adequados para disseminar a mensagem, assim como para alcançar o maior número de vizinhos. E também, o conceito de Computação Autonômica, que é utilizada para decisão de quando retransmitir uma mensagem de dados, baseado em uma eficiência de propagação calculada a partir do número de mensagens transmitidas e o número de beacons recebidos em cada veículo, fazendo com que o veículo saiba quando retransmitir ou manter a mensagem. O ALADDIN pode inserir overhead indesejado na rede ao armazenar várias mensagens quando um veículo identifica partição na rede (store-carry-forward) e encontra outro veículo capaz de continuar o processo de disseminação.

Cunha et al. [Cunha et al. 2014] introduziram o protocolo Clustering Coefficient and node DEGREE (CC-DEGREE), o qual identifica os melhores nós retransmissores com base em duas métricas: i) o coeficiente de agrupamento, o qual representa o número de conexões entre um veículo vizinho dividido pelo número total de conexões possíveis entre os vizinhos do veículo; ii) grau do nó, que representa o número de vizinhos a 1 salto que este nó possui. A partir dessas duas métricas, tempos para agendamento de retransmissão são atribuídos para veículos continuarem o processo de disseminação. O protocolo CC-DEGREE opera independente da densidade do tráfego. No entanto, o CC-DEGREE calcula o coeficiente de agrupamento baseado apenas na posição de cada veículo, resultando em uma baixa variabilidade para cenários densos e impactando a seleção dos nós retransmissores. Esse fato pode ocasionar na atribuição de tempos de espera muito próximos para vários veículos, aumentando assim as colisões no canal sem fio.

Akabane et al. [Akabane et al. 2016] apresentaram o protocolo de disseminação de dados em cenários urbanos e rodoviários denominado Context-Aware Routing pROtocol (CARRO). Tal protocolo explora o conhecimento do contexto geográfico para a 
disseminação em VANETs. O CARRO seleciona veículos localizados em setores geográficos de alta prioridade em seu raio de comunicação para continuar o processo de disseminação. Considera também, o mecanismo de store-carry-forward assim que o número de veículos na mesma área geográfica não é satisfatório para continuar o processo de disseminação. Cada veículo troca beacons periodicamente para obter informações de contexto sobre os veículos vizinhos a 1 salto, onde nos beacons são enviadas informações de direção, cenário em que o veículo se encontra, seu id e se o mesmo possui a mensagem. Entretanto, pelo fato do CARRO considerar o store-carry-forward para mitigar o problema de partição da rede, o atraso de entrega na disseminação de dados pode se elevar. Além disso, cada veículo também tem que transmitir o pacote em cada contato com veículos vizinhos que ainda não receberam este pacote, o que resulta em possível aumento de overhead.

Com base na análise dos trabalhos relacionados, conclui-se que é essencial estar ciente do conhecimento contextual além dos vizinhos de um salto sem aumentar o overhead. Isso otimiza a decisão de seleção do veículo retransmissor, permitindo obter uma alta cobertura com baixo overhead e atraso.

\section{Protocolo para Disseminação de Dados baseado em Métricas de Redes Complexas}

Esta seção descreve o DDRX, o qual considera uma abordagem distribuída baseada no emissor para selecionar os veículos retransmissores. Cada veículo deve manter o conhecimento local de seus vizinhos de até dois saltos, que é utilizado para construir um grafo e identificar o nó mais apropriado para continuar o processo de retransmissão. Vale ressaltar que o DDRX toma proveito dos beacons que naturalmente são trocados entre veículos com a adição de alguns campos específicos para seu funcionamento.

Definição I: Considere um cenário VANET composto por veículos equipados com um rádio compativel com o padrão IEEE 802.11p. Seja $G=(V(G), E(G))$ um grafo dinâmico que representa os enlaces sem fio assimétricos entre os veículos, onde $V(G)$ representa o conjunto de veículos e $E(G)$ representa o enlace de comunicação entre veículos vizinhos. Cada veículo $v \in V(G)$ conhece sua posição em andamento $p_{v}$ e o conjunto de veículos vizinhos $N_{v} \subseteq V(G)$ composto por vizinhos a 1 salto de $v$. Finalmente, seja $E_{v}^{\prime} \subseteq E(G)$ o conjunto de enlace de comunicação entre $v$ e seus vizinhos.

Na etapa de seleção de nós retransmissores, o DDRX considera duas métricas de redes complexas: i) centralidade de grau e ii) centralidade de intermediação. A centralidade de grau reflete a popularidade do vértice no grafo a partir do número de vizinhos [Daly and Haahr 2009]. Por outro lado, a métrica de centralidade de intermediação indica a importância de um dado vértice para o grafo em função da quantidade de caminhos mínimos que passam pelo mesmo [Cuzzocrea et al. 2012]. Em resumo, a intermediação está diretamente relacionada com o vértice cuja sua remoção desconecta a rede, ou seja, os vértices de corte $^{1}$ [Wang et al. 2016, Tian et al. 2017]. As métricas de centralidade são relevantes por quantificar o nível de importância que um vértice possui dentro da rede.

$\mathrm{Na}$ operação do protocolo DDRX, cada veículo $v$ deve transmitir beacons periódicos contendo sua identificação $i d$, sua posição atual $p_{v}$, e seus vizinhos de 1 salto $N_{v}$ para que cada veículo $v$ possa construir o conhecimento contextual sobre seus vizinhos. Ao receber um beacon, o veículo salva essa informação em sua lista de vizinhos

\footnotetext{
${ }^{1}$ Também conhecidos como Pontos de Articulação.
} 
$N_{v}$ e, no momento que necessita disseminar a mensagem, cria um subgrafo induzido por aresta $G\left[E_{u}^{\prime}\right]$ para cada $u \in N_{v}$, representando os enlaces de conexão entre o veículo $v$ e seus vizinhos de 1 e 2 saltos. Posteriormente, o protocolo DDRX seleciona os veículos para continuar o processo de disseminação de dados.

O DDRX identifica cada veículo candidato à retransmissor como um vértice de corte em cada $G\left[E_{u}^{\prime}\right]$ aplicando o algoritmo de Tarjan [Tarjan 1972]. Tal algoritmo faz uso da Busca em Profundidade (Dept-First-Search - DFS) para verificação desses vértices com complexidade no tempo $\mathcal{O}(V+E)$. Em alguns grafos simples, a remoção de um único vértice, chamado vértice de corte, faz com que o grafo se torne desconexo. Para entender, sejam $v, u$ e $x$ vértices em $G\left[E_{u}^{\prime}\right]$, onde $v$ só é considerado um vértice de corte se todo caminho entre $u$ e $x$ passa por $v$ ou existe um particionamento do conjunto de vértices $V-v$ em dois subconjuntos $V_{1}$ e $V_{2}$ tal que qualquer caminho entre $u \in V_{1} \mathrm{e}$ $x \in V_{2}$ passa obrigatoriamente pelo vértice $v$.

O algoritmo de Tarjan auxilia a encontrar os vértices com maior índice de centralidade de intermediação na rede. Sendo assim, cada veículo encontra um conjunto de vértices de corte com o algoritmo de Tarjan, que decompõe seu subgrafo $G\left[E_{u}^{\prime}\right]$ em uma DFS e aplica a função $\phi(v)$ para cada $v \in V\left(G\left[E_{u}^{\prime}\right]\right)$, conforme Equação 1.

$$
\phi(v)= \begin{cases}1 & v \text { é raiz e } N_{v} \geq 2 \\ 1 & v \text { não é raiz e possui um filho } u \\ & \text { de tal modo que } N_{v}-\{u\} \cap N_{u}-\{v\}=\emptyset \\ 0 & \text { Caso contrário }\end{cases}
$$

A Figura 1(a) apresenta o subgrafo $G\left[E_{u}^{\prime}\right]$ para o vértice $v$. Neste exemplo, o vértice $v$ pode identificar qual é o melhor candidato a ser selecionado como nó retransmissor. Neste caso são os vértices $u$ e $x$, uma vez que eles satisfazem as condições impostas no algoritmo de Tarjan. Onde, a remoção de $u$ e $x$ resultaria na desconexão direta de $s, t$ e $w$ com o restante do subgrafo, respectivamente.

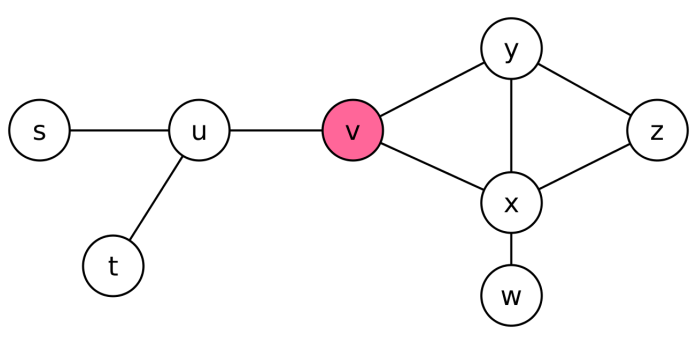

(a) Subgrafo Induzido por Arestas

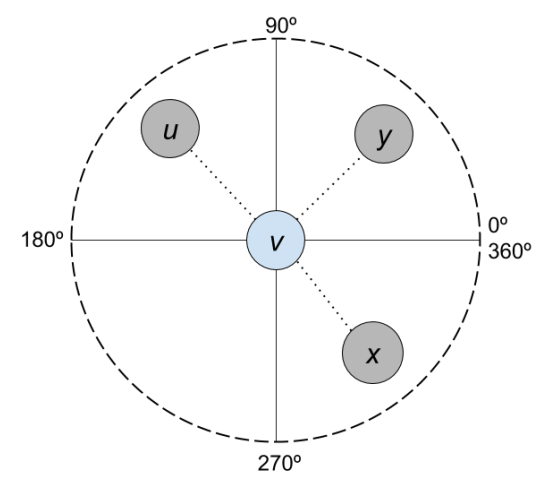

(b) Zonas de Encaminhamento

Figura 1. Operação do Protocolo DDRX

A utilização dessa abordagem para seleção é motivada pelo fato de que tais nós representam pontos comuns para troca de informações. Em outras palavras, o vértice de corte também é o vértice com o maior índice de centralidade de intermediação [Cuzzocrea et al. 2012, Freeman 1977]. Portanto, o DDRX diminui o número de trans- 
missões, uma vez que mais veículos serão alcançáveis através desses vértices selecionados com a adição do coeficiente de vizinhança, i.e., vértice com maior quantidade de vizinhos.

O DDRX seleciona o veículo que seja vértice de corte de acordo com a Equação 1, pois tal nó possui o maior coeficiente de vizinhança (ou seja, identificado a partir do maior índice de centralidade de grau) e que está mais próximo à borda do raio de comunicação para diminuir a quantidade de retransmissões da mensagem. Essa seleção ocorre com a decomposição do raio de comunicação do veículo $v$ em quatro quadrantes (ou seja, zonas de encaminhamento).

O protocolo DDRX verifica primeiramente se o veículo $v$ possui vértices de corte ou não. Caso positivo, identifica em qual zona de encaminhamento cada veículo candidato está com base em sua posição e posição já conhecida de seus veículos vizinhos candidatos, conforme ilustrado na Figura 1(b). O protocolo considera 4 zonas, ou seja, a primeiraZona $=[0,90]^{\circ}$ (p.e., vértice $y$ ), segundaZona $=[91,180]^{\circ}$ (p.e., vértice $u$ ), terceiraZona $=[181,270]^{\circ}$ (p.e., vértice $z$ ) ou quartaZona $=[271,359]^{\circ}$ (p.e., vértice $x$ ). Caso exista mais de um vértice de corte em uma única zona de encaminhamento, o DDRX calcula um ganho de retransmissão para cada um deles.

O calculo do ganho baseia-se em dois fatores principais: (i) distância relativa entre o veículo $v$ que está com a mensagem e seu vizinho $u$, a partir das posições $p_{v}$ conhecidas por cada um, tendo como limiar o raio de comunicação raio dos veículos; e ( ii) coeficiente de vizinhança, que indica o número de vizinhos $\left|N_{v}\right|$ (centralidade de grau) do veículo $u$ candidato à retransmissor dividido pelo maior grau de vizinhança $\max \left(N_{v}\right)$ em $G\left[E_{u}^{\prime}\right]$. Todos esses parâmetros são multiplicados por um valor $w$ para manter o resultado no intervalo [0,1], conforme denotado na Equação 2.

$$
\underset{v \in V\left(G\left[E_{u}^{\prime}\right]\right) \mid \phi(v)=1}{\arg \max } w\left(\frac{\operatorname{distancia}(u, v)}{\operatorname{raio}}+\frac{\left|N_{v}\right|}{\max \left(N_{v}\right)}\right)
$$

Caso não seja identificado pelo menos um vértice de corte após verificação através da Equação 1, o protocolo DDRX deve selecionar o veículo que possui o maior coeficiente de vizinhança em cada zona de encaminhamento e que também está mais próximo do raio de comunicação de $v$. Considera-se essa abordagem pelo fato de que quanto mais vizinhos um veículo possui (maior centralidade de grau), mais veículos à 1 salto recebem sua mensagem [Cunha et al. 2014].

Após identificação dos nós retransmissores, o protocolo DDRX adiciona os seus respectivos $i d s$ na mensagem e a envia em broadcast. Conforme recebimento da mensagem, cada receptor verifica se a mesma já foi recebida anteriormente para evitar retransmissão redundante. Se já a recebeu, descarta. Caso o veículo ainda não tenha recebido, verifica-se a informação dos retransmissores selecionados que está contida na mensagem. Se o veículo receptor possuir o mesmo $i d$ indicado na mensagem, ele inicia um novo processo de seleção conforme indicado na Equação 1 a partir de seu $G\left[E_{u}^{\prime}\right]$ construído com as informações de troca de beacons com seus vizinhos.

\section{Avaliação}

Esta seção descreve a metodologia e métricas utilizadas para avaliar a eficiência do protocolo DDRX em termos de cobertura, transmissões, atraso e colisões. Foram realizadas simulações com diferentes densidades para analisar a eficiência do protocolo DDRX comparado aos protocolos CARRO, CC-DEGREE, UV-CAST e Flooding. 


\subsection{Metodologia}

As simulações foram realizadas no framework $\mathrm{VEINS}^{2}$ do OMNeT $++^{3}$, o qual implementa a pilha de protocolo do padrão IEEE 802.11p para comunicação V2V e atenuação de sinal. Para estabelecer um cenário de avaliação, utilizou-se dez ruas de duas pistas em uma área de $1 \mathrm{Km}^{2}$ da cidade de Manhattan nos Estados Unidos. Essa região foi obtida através do OpenStreetMap ${ }^{4}$ e importada pelo Simulation of Urban MObility (SUMO) ${ }^{5}$ para gerar os registros de movimentação dos veículos. Isso permite reproduzir os movimentos dos veículos e as interações $\mathrm{V} 2 \mathrm{~V}$, de acordo com dados empíricos.

Os efeitos de atenuação do sinal causados por edifícios foram considerados, onde assumiu-se que cada bloco possui um obstáculo de $80 \mathrm{~m}$ x $80 \mathrm{~m}$ para representação de edifícios altos. Para quantificar a evolução do tráfego neste cenário, variou-se a densidade de veículos em 100, 200, 300, 400, 500, 600 e 700 veículos $/ \mathrm{km}^{2}$, com uma área de interesse de $1 \mathrm{Km}^{2}$. A velocidade dos veículos respeita os limites impostos pelo cenário, sendo de no máximo $50.04 \mathrm{~km} / \mathrm{h}$ em cada via.

A taxa de bits na camada MAC foi definida em 18 Mbit/s e a potência de transmissão para $2.2 \mathrm{~mW}$. Esses parâmetros, juntamente com o modelo de propagação Two-Ray ground, fornecem um raio de comunicação de 300 metros. A frequência de envio de beacons foi de $1 \mathrm{~Hz}$. Cada simulação foi executada 33 vezes com diferentes sementes de aleatoriedade e os resultados apresentam os valores com um intervalo de confiança de 95\%. O tempo de simulação foi de 150 segundos, que é suficiente para avaliar os algoritmos de disseminação [Meneguette et al. 2014].

No momento da simulação em que a maioria dos veículos estavam no cenário, um veículo foi selecionado para dar início ao processo de disseminação a partir do envio de uma única mensagem de dados com tamanho de 2048 bytes. O veículo selecionado é o que esta mais próximo ao centro do cenário. A Tabela 1 resume os parâmetros utilizados na avaliação.

Tabela 1. Parâmetros de Simulação para o Cenário de Manhattan Grid

\begin{tabular}{l|l}
\hline Parâmetro & Valor \\
\hline Tempo de simulação & $150 \mathrm{~s}$ \\
Densidade de veículos & de 100 a 700 veículos $/ \mathrm{km}^{2}$ \\
Tamanho do cenário & $1 \mathrm{Km}^{2}$ \\
Área de interesse & $1 \mathrm{Km}^{2}$ \\
Potência de transmissão & $2.2 \mathrm{~mW}$ \\
Raio de comunicação & $300 \mathrm{~m}$ \\
Taxa de bits & $18 \mathrm{Mbits} / \mathrm{s}$ \\
Frequência de beacons & $1 \mathrm{~Hz}$ \\
Tamanho da mensagem de dados & 2048 bytes \\
\hline
\end{tabular}

Foram consideradas as seguintes métricas para a avaliação: (i) Cobertura: reflete a porcentagem de veículos dentro da AoI que recebem a mensagem; (ii) Número de mensagens transmitidas: total de transmissões da mensagem, usado para medir o overhead;

\footnotetext{
${ }^{2}$ http: //veins.car2x.org/

${ }^{3}$ http: / / omnetpp.org/

${ }^{4}$ http: //www.openstreetmap.org/

${ }^{5}$ http: //sumo.dlr.de
} 
(iii) Atraso: atraso médio de entrega da mensagem da fonte para todos os veículos dentro da AoI; e (iv) Colisões: número total de colisões de pacotes na camada MAC.

\subsection{Análise dos Resultados}

A Figura 2(a) apresenta a cobertura atingida pelos protocolos DDRX, CARRO, UVCAST, CC-DEGREE e Flooding. Ao analisar os resultados observa-se que todos os protocolos atingem pelo menos $94.8 \%$ de cobertura em densidade mais baixa e acima de $98 \%$ em densidade mais alta. O protocolo CC-DEGREE mesmo na menor densidade atinge o melhor desempenho com $96.8 \%$ de cobertura, mas conforme a densidade aumenta sua cobertura decai em relação ao protocolo CARRO. O CARRO tem $96.6 \%$ de cobertura na menor densidade e supera os demais protocolos na maior densidade, com $98.8 \%$ de cobertura. O protocolo DDRX destaca-se por aumentar sua cobertura conforme aumento da densidade, ou seja, aumenta de $94.8 \%$ para $98.4 \%$. A menor cobertura do DDRX em comparação aos demais protocolos nas densidades mais baixas é justificado pelo fato de que os protocolos UV-CAST, CC-DEGREE e CARRO consideram o mecanismo de store-carry-forward para garantia de entrega das mensagens. As altas taxas de cobertura alcançadas pelo Flooding são reflexo da falta de seleção de nós retransmissores, impactando no aumento do número de transmissões tal como apresentado na Figura 2(b).

A Figura 2(b) demonstra o número de transmissões que cada protocolo necessita para disseminar dados na rede. Como pode ser observado, o DDRX diminui o número de transmissões em $90.25 \%, 64.10 \%, 57.12 \%$ e $48.92 \%$ em relação aos protocolos Flooding, CARRO, UV-CAST e CC-DEGREE, respectivamente. Isso é resultado da seleção de nós retransmissores em cada zona de encaminhamento realizada pelo protocolo DDRX. Os protocolos CARRO, CC-DEGREE e UV-CAST consideram a transmissão do pacote em cada contato com um veículo vizinho que ainda não recebeu este pacote, aumentando assim o número de transmissões e atraso tal como apresentado na Figura 2(c). O elevado número de transmissões para o Flooding é decorrente da não seleção de nós retransmissores, ou seja, todos veículos que recebem a mensagem de dados a retransmitem. Tanto CC-DEGREE quanto DDRX utilizam métricas de redes complexas e/ou teoria dos grafos para selecionar nós retransmissores, justificando porque tais protocolos obterem o menor número de transmissões. Vale ressaltar que é essencial realizar a disseminação de dados com baixo número de transmissões para não afetar o desempenho de outras aplicações que compartilham o mesmo canal sem fio.

A Figura 2(c) exibe o atraso médio para entrega de dados pelos protocolos DDRX, CARRO, UV-CAST, CC-DEGREE e Flooding. O protocolo DDRX dissemina a mensagem com $89.27 \%, 84.32 \%, 41.56 \%$ e $40.97 \%$ menos atraso que os protocolos CCDEGREE, UV-CAST, CARRO e Flooding, respectivamente. Os protocolos UV-CAST e CC-DEGREE têm maiores atrasos em comparação com os outros protocolos tanto em cenários esparsos quanto densos, uma vez que consideram o mecanismo de store-carryforward. O CARRO mesmo considerando o store-carry-forward obtém um baixo atraso entre 0.07 e 0.11 segundos por não depender exclusivamente do store-carry-forward para verificação de entrega da mensagem. O protocolo DDRX obteve atraso médio de 0.05 segundos. O atraso é uma métrica crucial para certos tipos de aplicação, tal como um TMS [de Souza et al. 2017b], pois as decisões podem ser tomadas mais rapidamente.

A Figura 2(d) apresenta o número médio de colisões de pacotes na camada MAC. Essa métrica permite observar como os protocolos lidam com o aspecto distribuído em seu funcionamento, pois se dois ou mais transmitem uma mensagem com tempos próximos 
elas irão colidir e a mensagem será perdida, resultando em retransmissões. Como esperado, o Flooding é o protocolo com maior número de colisões, sendo resultado da não coordenação no processo de retransmissão de pacotes, uma vez que muitos veículos tentam acessar o canal ao mesmo tempo. O protocolo DDRX possui em média $90.71 \%$, $53.71 \%$, 46.37\% e $32.77 \%$ menos colisões que os protocolos Flooding, UV-CAST, CCDEGREE e CARRO, respectivamente. Isso deve-se a seleção de nós retransmissões eficiente efetuada pelo DDRX, onde diminui-se a quantidade de candidatos a partir da identificação dos vértices de corte em cada zona de encaminhamento, e também pela atribuição de uma distribuição uniforme no momento em que os poucos veículos selecionados agendam suas transmissões.

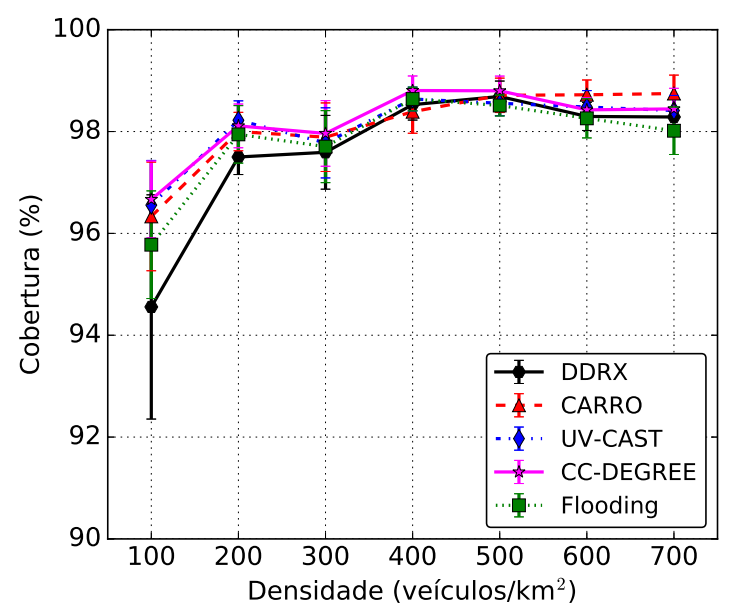

(a) Cobertura

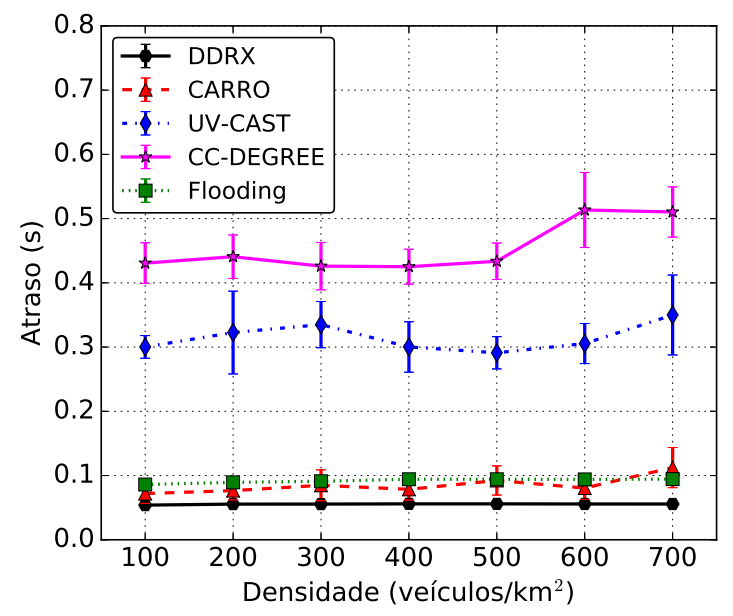

(c) Atraso

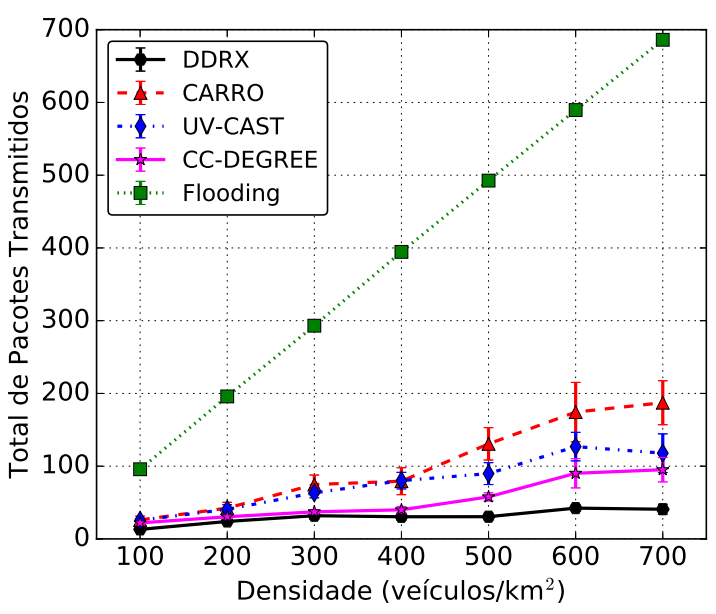

(b) Número de Mensagens Transmitidas

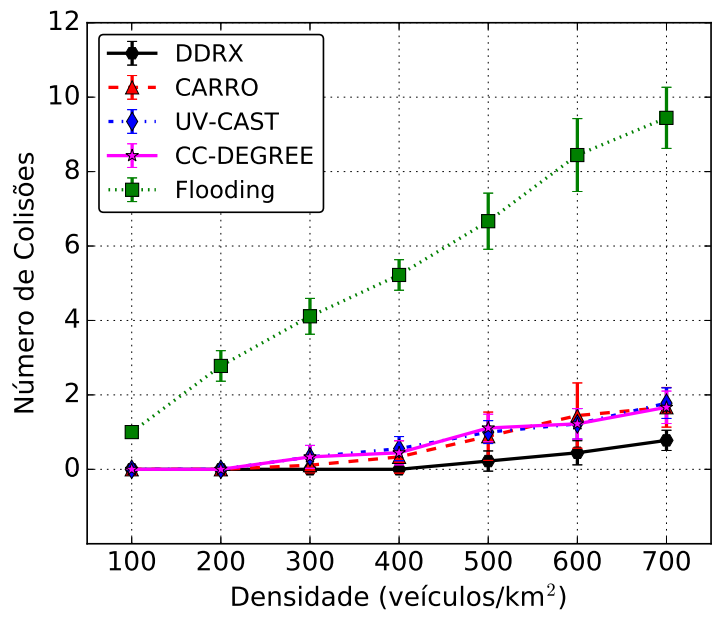

(d) Número de Colisões

Figura 2. Resultados de Simulação para Disseminação de Dados no Cenário de Manhattan Grid

\section{Estudo de Caso: Protocolo DDRX aplicado em um TMS}

O crescimento desproporcional da quantidade de veículos em relação às melhorias da infraestrutura viária acarreta na incidência de alguns problemas nos grandes centros urbanos [Cunha et al. 2017]. Um desses problemas é o congestionamento, onde muita das vezes os condutores não têm nenhum suporte adicional para evitar ou minimizar os impactos 
desse tipo de situação. Dessa forma, os TMSs têm como objetivo maximizar a eficiência da infraestrutura de transporte disponível minimizando os congestionamentos e todos danos à eles relacionados [de Souza et al. 2017a]. Desta forma, esta seção apresenta um estudo de caso para validar o funcionamento do protocolo DDRX em conjunto com uma aplicação de TMS, a qual necessita da disseminação de dados eficiente para operação.

Para este caso de uso foi considerado o TMS denominado FASTER [de Souza and Villas 2016], o qual tem como características principais ser totalmente distribuído e segmentar o cenário em diferentes distritos (i.e., subregiões) para poder agregar as informações de tráfego das vias. No FASTER, um distrito tem tamanho igual ao raio de comunicação dos veículos. Cada veículo coleta e transmite informações de velocidade média e identificação das vias em seu raio de comunicação através de beacons, onde as vias que estiverem por completo são denominadas totalmente cobertas e as que não estiverem são denominadas parcialmente cobertas. Na operação do FASTER, quando um veículo recebe beacons com informações das vias, o mesmo armazena essa informação em suas listas de conhecimento de tráfego. O veículo atualiza a informação caso possua uma entrada, e caso contrario adiciona a informação em sua lista. O veículo que estiver mais próximo ao centro do distrito inicia o processo de envio das informações de tráfego agregadas para que outros veículos também atualizem suas listas de conhecimento a fim de criar um conhecimento local do distrito. Nesta etapa, tem-se a primeira disseminação de dados realizada no FASTER.

Em seguida, como as informações de tráfego já foram disseminadas no interior de cada distrito, faz-se necessário disseminar essas informações para os demais distritos, construindo assim um conhecimento global acerca das condições de tráfego. É então que outra disseminação se inicia pelo veículo que estiver mais próximo ao centro de cada distrito, onde é feita uma verificação para que apenas veículos de outros distritos retransmitam a mensagem para não gerar redundâncias para os que já receberam as informações do distrito na primeira disseminação. O FASTER divide a disseminação de dados em duas para não ocasionar overhead indesejado na rede com a criação dos conhecimentos de tráfego. Desta forma, este estudo de caso esta focado na aplicação do DDRX para eliminar a primeira disseminação de dados, ou seja, a de criação do conhecimento em cada distrito, fazendo com que a disseminação do conhecimento de tráfego seja enviada para todo o cenário de uma só vez.

Sendo assim, o DDRX foi integrado ao FASTER utilizando as ferramentas supracitadas na Seção 4. Também foi considerada uma região de $1 \mathrm{Km}^{2}$ do centro da cidade de Manhattan, extraída com o OpenStreepMaps. Os veículos foram inseridos no cenário de forma que a densidade de veículos atinga 500, 700, 900 e 1100 veículos $/ \mathrm{km}^{2}$, onde nessas densidades existem congestionamentos. Cada simulação foi executada 33 vezes com diferentes sementes de aleatoriedade e os resultados apresentam os valores com um intervalo de confiança de $95 \%$. O tempo de simulação foi de 2000 segundos. Utilizouse as informações de mobilidade do cenário sem aplicação de nenhum mecanismo de TMS como baseline, denominadas aqui como Mobilidade Original do Cenário (MOC). Foi utilizado o FASTER original, e o FASTER considerando o DDRX como forma de disseminação (FASTER-DDRX). Desta forma, para esta avaliação verificou-se o comportamento de MOC, FASTER e FASTER-DDRX em termos de número de mensagens transmitidas para criação do conhecimento (quando se aplica), tempo médio de viagem, tempo médio de congestionamento e velocidade média atingida pelos veículos.

A Figura 3(a) exibe o número de transmissões realizadas para gerenciamento de 
trânsito pelo FASTER e FASTER-DDRX. Pode-se observar que o FASTER-DDRX transmite cerca de $95 \%$ menos mensagens que o FASTER. Isso deve-se pela eliminação da disseminação de dados para criar o conhecimento entre os distritos. Ou seja, o FASTERDDRX consegue disseminar em todo o cenário as informações de tráfego das vias inserindo um menor overhead na rede e sem comprometer o bom funcionamento do FASTER.

A Figura 3(b) apresenta o tempo médio que os veículos levam para concluírem suas viagens considerando o FASTER, FASTER-DDRX e MOC. Nesta avaliação, podese observar que tanto na densidade de 700 quanto na de 1100 veículos $/ \mathrm{km}^{2}$, a aplicação do FASTER resulta em maior tempo de viagem em comparação a MOC e FASTER-DDRX. Por outro lado, na densidade de 700 veículos o FASTER-DDRX diminui em $1.13 \%$ o tempo médio de viagem em relação aos demais, tendo um menor tempo de viagem mais perceptível para densidade acima de 900 veículos, sendo menor $18.11 \%$ que os demais. O aumento no tempo de viagem pode ser resultado do reencaminhamento dos veículos, onde as rotas originais são substituídas por rotas mais longas com menor incidência de congestionamentos tal como pode ser observado na Figura 3(c).

A Figura 3(c) exibe o tempo médio que os veículos ficam parados em congestionamentos. Esta avaliação é importante pois mostra a eficiência do algoritmo de roteamento de veículos em relação às condições de tráfego veicular identificadas. Pode-se perceber que com o FASTER-DDRX os veículos ficam menos tempo em congestionamentos. Nas densidades de 500, 700 e 900 veículos/ $\mathrm{km}^{2}$ o FASTER-DDRX tem resultados próximos ao FASTER, e ambos possuem menor tempo de congestionamento quando comparados a MOC. Já na densidade de 1100 veículos $/ \mathrm{km}^{2}$, o FASTER-DDRX tem resultados superiores a MOC e FASTER. Isso se justifica pela disseminação eficiente empregada no DDRX, especialmente quando a rede está mais densa e a seleção de nós retransmissores possui mais opções de candidatos. Essa análise apresenta que FASTER-DDRX possui desempenho semelhante e até superior ao FASTER, em alguns casos, reiterando a eficiência de disseminação do protocolo DDRX.

A Figura 3(d) exibe a velocidade média atingida pelos veículos considerando o FASTER, FASTER-DDRX e MOC. Nesta avaliação, velocidades médias superiores são indicativos de que os veículos não foram roteados para vias com alto índice de congestionamentos. Sendo assim, pode-se notar que o FASTER-DDRX atinge maiores índices de velocidade em todas as densidades, a qual decai conforme a densidade aumenta devido os maiores índices de congestionamentos. O FASTER se mantém superior ao MOC em todas as densidades, pois reencaminha os veículos eficientemente para rotas menos congestionadas. As rotas que foram indicadas pelo FASTER-DDRX possuem menores índices de tráfego fazendo com que os veículos atinjam maiores velocidades e, consequentemente, percam menos tempo em congestionamentos, conforme já apresentado na Figura 3(c).

A partir da análise dos resultados, identificou-se que o FASTER-DDRX reduz em 95\% a quantidade de mensagens disseminadas na rede para criação do conhecimento global de tráfego, em relação ao FASTER. O FASTER-DDRX mantém tempo de viagem menor que MOC e FASTER, sendo de $18.11 \%$, e as rotas encontradas para os veículos tiveram o menor índice de congestionamentos, ou seja, congestionamento $48.95 \%$ e $20.5 \%$ menor em relação ao MOC e FASTER, respectivamente. Isso impacta diretamente no aumento geral da velocidade média atingida pelos veículos com o FASTER-DDRX, tendo aumento de $8 \%$ e $9 \%$ em relação a FASTER e MOC, respectivamente. 


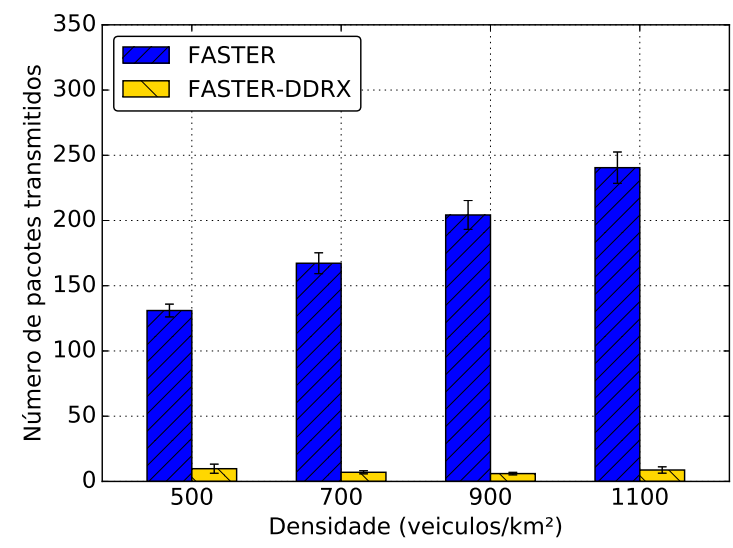

(a) Número de Transmissões

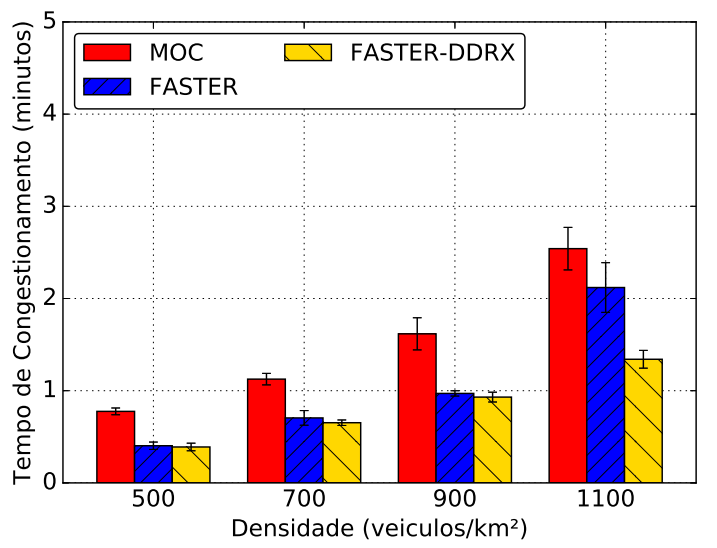

(c) Tempo em Congestionamento

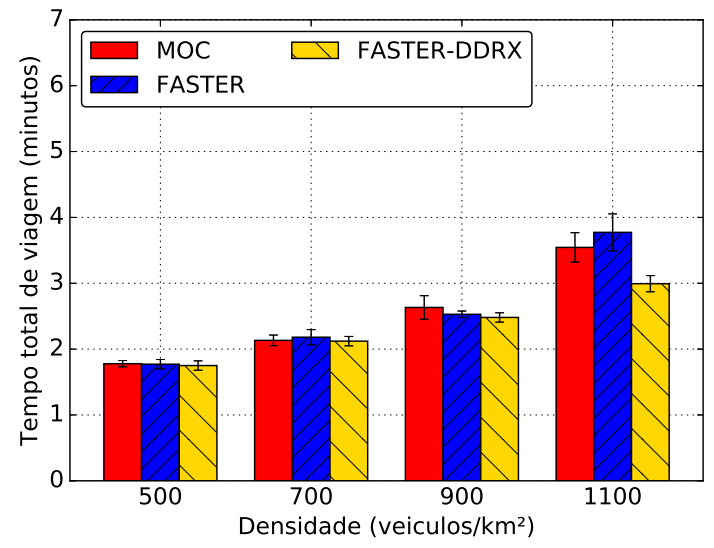

(b) Tempo Total de Viagem

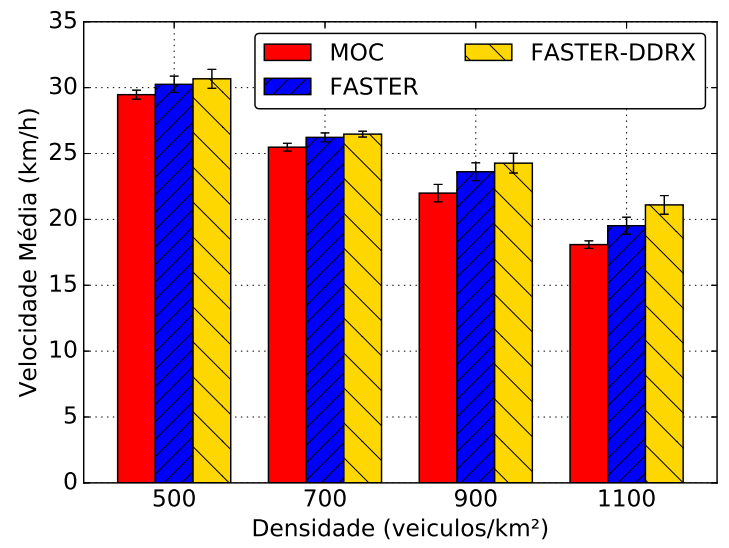

(d) Velocidade

Figura 3. Resultados de Simulação para Disseminação de Dados de TMS

\section{Conclusão}

Aplicações em VANETs necessitam que os dados sejam disseminados na rede para execução de suas tarefas. Porém, esta é uma tarefa desafiadora devido as características específicas das VANETs, tais como, mobilidade altamente dinâmica, tempo curto de contato entre os veículos e comunicação de curto alcance. Desta forma, foi apresentado o protocolo DDRX para disseminação de dados com baixo overhead, colisões e atraso, mantendo a alta cobertura. No DDRX, cada veículo deve manter o conhecimento local de seus vizinhos de 1 e 2 saltos, que serão usados para construção de um grafo de diâmetro 2. Com o grafo criado, métricas de redes complexas são utilizadas a fim de identificar os melhores veículos para retransmitirem a mensagem.

Foram realizadas simulações com os protocolos DDRX, UV-CAST, CCDEGREE, CARRO e Flooding. Os resultados obtidos mostraram que o DDRX reduz o atraso, overhead e colisões, mantendo uma boa cobertura. Adicionalmente, avaliou-se o DDRX operando em conjunto com um TMS, denominado FASTER-DDRX, que necessita de uma disseminação de dados eficiente para tomar decisões acerca do gerenciamento de tráfego. Os resultados mostraram que o DDRX aplicado ao TMS não compromete o bom funcionando do TMS e, em alguns casos, implica em um melhor gerenciamento de tráfego. Como trabalhos futuros, pretende-se avaliar o DDRX em cenários maiores, avaliar o FASTER-DDRX com outras métricas de gerenciamento de tráfego e utilizar os 
conceitos do DDRX para criação de um TMS que se baseia inteiramente em métricas de redes complexas.

\section{Agradecimentos}

Os autores gostariam de agradecer o apoio financeiro concedido pela Coordenação de Aperfeiçoamento de Pessoal de Nível Superior (CAPES), pela Fundação de Amparo à Pesquisa do Estado de São Paulo (FAPESP) através dos processos $n^{\circ}$ 2015/07538-1 e 2015/24494-8 e ao Conselho Nacional de Desenvolvimento Científico e Tecnológico (CNPq) através do processo ${ }^{\circ}$ 401802/2016-7.

\section{Referências}

Akabane, A., Pazzi, R., Madeira, E., and Villas, L. A. (2016). CARRO: A ContextAwareness Protocol for Data Dissemination in Urban and Highway Scenarios. In Proceedings of the 8th IEEE Latin-American Conference on Communications (LATINCOM'16), pages 1-6. IEEE.

Alvarenga, D., da Cunha, F. D., Viana, A. C., Mini, R. A., and Loureiro, A. A. (2014). Classificando comportamentos sociais em redes veiculares. In Anais do XXXIV Simpósio Brasileiro de Redes de Computadores e Sistemas Distribuídos (SBRC'14).

Cunha, F., Maia, G., Celes, C., Guidoni, D., de Souza, F., Ramos, H., and Villas, L. A. (2017). Sistemas de transporte inteligentes: Conceitos, aplicações desafios. In Livro de Minicursos do Simpósio Brasileiro de Redes de Computadores e Sistemas Distribuídos (SBRC'17).

Cunha, F., Maia, G., Viana, A., Mini, R., Villas, L. A., and Loureiro, A. (2014). Socially inspired data dissemination for vehicular ad hoc networks. In Proceedings of the 17th ACM international conference on Modeling, analysis and simulation of wireless and mobile systems, pages 81-85. ACM.

Cunha, F., Villas, L. A., Boukerche, A., Maia, G., Viana, A., Mini, R., and Loureiro, A. (2016). Data communication in vanets: Protocols, applications and challenges. Ad Hoc Networks, 44:90-103.

Cuzzocrea, A., Papadimitriou, A., Katsaros, D., and Manolopoulos, Y. (2012). Edge betweenness centrality: A novel algorithm for qos-based topology control over wireless sensor networks. Journal of Network and Computer Applications, 35(4):12101217.

Daly, E. M. and Haahr, M. (2009). Social network analysis for information flow in disconnected delay-tolerant manets. IEEE Transactions on Mobile Computing, 8(5):606-621.

de Souza, A. M., Botega, L. C., and Villas, L. A. (2017a). GTE: Um Sistema para Gerenciamento de Trânsito Escalável baseado em Compartilhamento Oportunista. In Anais do Simpósio Brasileiro de Redes de Computadores e Sistemas Distribuídos (SBRC'17).

de Souza, A. M., Brennand, C. A., Yokoyama, R. S., Donato, E. A., Madeira, E. R., and Villas, L. A. (2017b). Traffic management systems: A classification, review, challenges, and future perspectives. International Journal of Distributed Sensor Networks, 13(4):1-14.

de Souza, A. M. and Villas, L. A. (2016). A Fully-distributed Traffic Management System to Improve the Overall Traffic Efficiency. In Proceedings of the 19th ACM Internatio- 
nal Conference on Modeling, Analysis and Simulation of Wireless and Mobile Systems (MSWiM'16), pages 19-26.

de Souza, A. M., Yokoyama, R. S., Maia, G., Loureiro, A., and Villas, L. A. (2016). Real-time Path Planning to Prevent Traffic Jam Through an Intelligent Transportation System. In Proceedings of the IEEE Symposium on Computers and Communication (ISCC'16), pages 726-731. IEEE.

Freeman, L. C. (1977). A set of measures of centrality based on betweenness. Sociometry, pages $35-41$.

Gerla, M., Wu, C., Pau, G., and Zhu, X. (2014). Content Distribution in VANETs. Vehicular Communications, 1(1):3-12.

Lv, Y., Duan, Y., Kang, W., Li, Z., and Wang, F.-Y. (2015). Traffic flow prediction with big data: a deep learning approach. IEEE Transactions on Intelligent Transportation Systems, 16(2):865-873.

Meneguette, R. I., Maia, G., Madeira, E. R., Pinto, A. R., Loureiro, A. A., and Villas, L. A. (2014). Um algoritmo autônomo para disseminação de informações em redes veiculares. In Anais do Simpósio Brasileiro de Redes de Computadores e Sistemas Distribuídos (SBRC'14).

Newman, M. E. (2003). The structure and function of complex networks. SIAM review, 45(2):167-256.

Silva, F., Boukerche, A., Silva, T., Ruiz, L., Cerqueira, E., and Loureiro, A. (2016). Vehicular networks: a new challenge for content-delivery-based applications. $A C M$ Computing Surveys (CSUR), 49(1):11.

Tarjan, R. (1972). Depth-first search and linear graph algorithms. SIAM journal on computing, 1(2):146-160.

Tian, L., Bashan, A., Shi, D.-N., and Liu, Y.-Y. (2017). Articulation points in complex networks. Nature Communications, 8.

Viriyasitavat, W., Bai, F., and Tonguz, O. (2010). UV-CAST: an Urban Vehicular Broadcast Protocol. In Proceedings of the IEEE Vehicular Networking Conference (VNC'10), pages 25-32. IEEE.

Wang, J., Jiang, C., Zhang, K., Quek, T. Q., Ren, Y., and Hanzo, L. (2017). Vehicular sensing networks in a smart city: Principles, technologies and applications. IEEE Wireless Communications.

Wang, L., Yang, F., Zhuang, L., Cui, H., Lv, F., and Feng, X. (2016). Articulation points guided redundancy elimination for betweenness centrality. In Proceedings of the 21st ACM SIGPLAN Symposium on Principles and Practice of Parallel Programming, page 7. ACM.

Wang, M., Shan, H., Lu, R., Zhang, R., Shen, X., and Bai, F. (2015). Real-time Path Planning based on Hybrid-VANET-enhanced Transportation System. IEEE Transactions on Vehicular Technology, 64(5):1664-1678.

Zhang, H. and Li, J. (2015). Topology analysis of vanet based on complex network. In LISS 2014, pages 1143-1148. Springer. 